\title{
Adhesive Strength and Toughness Improvement of Epoxy Resin Modified with Polystyrene-B-Polybutadiene-B-Poly (Methyl Methacrylate) Block Copolymer
}

\author{
SG Prolongo ${ }^{1 *}$, MR Gude ${ }^{2}$ and A Urena
}

${ }^{1}$ Departmentof Materials Science and Engineering, University Rey Juan Carlos, Madrid, Spain

${ }^{2}$ Foundation for Research, Development and Application of Composite Materials (FIDAMC) Avda. Rita Levi Montalcini, Madrid, Spain

\begin{abstract}
Polystyrene-b-polybutadiene-b-poly (methyl methacrylate) block copolymer (SBM) was incorporated into epoxy resin to access the nanostructures in epoxy thermosets, knowing the different miscibility of polymeric blocks on epoxy matrix during the curing treatment. The morphology of modified SBM/epoxy resins was examined by Transmission and Field Emission Gun Scanning Electron Microscopy (TEM and FEG-SEM), reveling a nanostructured epoxy matrix with a dispersed micro-scale phase. The modified resins presented enhanced flexural properties, which were dependent on the composition of blend. The resin reinforced with low SBM contents (2.5 and 5 wt\%) presented high values of flexural modulus and strength, while the blend reinforced with higher SBM load (10 wt\%) showed higher elongation ability. This different behavior was explained by SEM analysis of fracture surfaces, which showed different toughening mechanisms. The adhesive strength and toughness were determined using carbon fiber/epoxy laminates with peel ply surface treatment as adherends. The results showed a dramatic enhancement of both properties for modified SBM/epoxy adhesives. The highest increases measured were 50 and $70 \%$ for lap shear strength and mode-I adhesive fracture energy, respectively.
\end{abstract}

Keywords: Nanostructured materials; Bonding; Electron microscopy; Mechanical characterization

\section{Introduction}

Epoxy resins are one of the most important structural adhesives due to their good adhesive properties and high thermal and chemical strength. These adhesives show good wettability on carbon fibre/epoxy laminates due to the high compatibility with epoxy matrix. For this reason, they are being actually investigated in order to replace the mechanical joints. The adhesive joint of epoxy laminates is especially interesting for automotive and aerospace industry, which need large and complex structures combined with a low weight.

The main limitation of epoxy adhesives is their low fracture toughness. In the last two decades, numerous works has been undertaken in an attempt to enhance their toughness. The main approaches are based on the incorporation of a second phase, such as rubber or thermoplastic particles and mineral fillers [1-3]. It is well known that relative low corsslinked epoxy thermoset can be toughened by incorporation of elastomeric micro-phases but high crosslinked resins are difficult to toughen by this procedure [4,5]. Also, the addition of elastomeric polymers generally tends to decrease both the elastic modulus and heat resistance. Therefore, new engineering thermoplastic polymers and copolymers are being researched to achieve high toughness and thermal strength. Block copolymers have attracted attention as modifiers for toughening of epoxy resins [6,7]. These tend to form nano-phase structures (1-100 nm). In general, the efficiency of fillers is inversely proportional to size and directly proportional to the filler surface area and volume ratio [6].

The enhancement in toughness depended on the phase-separated morphology of modified resins. The thermosetting blends are usually prepared from homogeneous solution composed of epoxy precursors and thermosetting modifier. The phase separation occurs due to the change of free energy in the mixture elevated by the increase in the molecular weight during epoxy curing reaction (Reaction-induced phase separation, RIPS) [2]. The size of separated phases is in the order of sub-micrometers or nanometers [8-10]. When the thermoplastic modifiers are homopolymers or random copolymers, the reactioninduced phase separation occurs on microscopic scale. The use of block copolymer with miscible block allows obtaining nano-scale inclusions or even nano-structures.

Ritzenthaler et al. [11-14] studied the addition of ABC triblock copolymers to epoxy resins. The used thermoplastic modifier was polystyrene-b-polybutadiene-b-poly (methyl methacrylate) (PS-PBPMMA, SBM). The morphology of blends markedly depended on the characteristic of thermoplastic copolymer and even the nature of hardener. For example, the increase of PB content made "onion like" multilayer morphology. It was determined that PS and PB blocks are immiscible while PMMA is completely miscible. The addition of low amount of SBM can induce an important enhancement of toughness of epoxy resin. The major toughening mechanism implied is shear yielding although other mechanism can be implied such as particle bridging mechanism, crack-pinning or microcracking.

The purpose of this work is to investigate the viability of using toughened epoxy resins, formed by thermoset matrix with different content of SBM, as adhesives for joining carbon fiber/epoxy laminates. The objective is to increase the toughness of joints through the presence of new micromechanical mechanisms associated to the presence of different thermoplastic nano- and micro-scale phases that contribute to increase the energy consumption during fracture.

*Corresponding author: SG Prolongo, Department of Materials Science and Engineering, University Rey Juan Carlos, Madrid, Spain, E-mail: silvia.gonzalez@urjc.es

Received July 25, 2012; Accepted August 21, 2012; Published August 28, 2012

Citation: Prolongo SG, Gude MR, Urena A (2012) Adhesive Strength and Toughness Improvement of Epoxy Resin Modified with Polystyrene-BPolybutadiene-B-Poly (Methyl Methacrylate) Block Copolymer. J Material Sci Eng 1:109. doi:10.4172/2169-0022.1000109

Copyright: (c) 2012 Prolongo SG, et al. This is an open-access article distributed under the terms of the Creative Commons Attribution License, which permits unrestricted use, distribution, and reproduction in any medium, provided the original author and source are credited. 
Citation: Prolongo SG, Gude MR, Urena A (2012) Adhesive Strength and Toughness Improvement of Epoxy Resin Modified with Polystyrene-BPolybutadiene-B-Poly (Methyl Methacrylate) Block Copolymer. J Material Sci Eng 1:109. doi:10.4172/2169-0022.1000109

\section{Experimental}

\section{Materials}

The unidirectional carbon fibre/epoxy laminates used as adherends are designed for use in primary aerospace structures. They were manufactured by INTA (Madrid, Spain), from unidirectional prepregs (Hexply 8552/34\%/UD134/AS4-12K), supplied by Hexcel (Stamford, USA) laid up and then cured in an autoclave at $180^{\circ} \mathrm{C}$ for $2 \mathrm{~h}$ at a pressure of 6 bar. The nominal fibre volume is 57\%. A dry polyester peelply (Release Ply C, Airtech (Differdange, Luxembourg) was placed over the last prepreg. This ply was removed just before bonding, to generate a rough surface free of contamination. The composite surface has been characterized by measurements of roughness and surface free energy in a previous work.

The epoxy adhesive is constituted by diglycidyl ether of bisphenol A (DGEBA), with 178 g/epoxy equivalent, and 4,4-diamino diphenyl methane (DDM) as curing agent. Both components were supplied by Sigma-Aldrich. The SBM triblock copolymer was manufactured by Nanostrength from Arkema (France) with the commercial name E41 [15]. It is formed by polystyrene, 1,4-polybutadiene and syndiotactic poly (methyl methacrylate) and self-organizes on a nanometer scale induced by the strong repulsions between the side and middle blocks. The own manufacturer indicates that the addition of $5-15 \mathrm{wt} \% \mathrm{SBM}$ to epoxy resin (DGEBA/DDM) induces an increase of toughness from 0.74 to $1.08 \mathrm{MPa}^{0.5}$ in $\mathrm{K}_{\mathrm{Ic}}$ and from 258 to $418 \mathrm{~J} / \mathrm{m}^{2}$ in $\mathrm{G}_{\mathrm{Ic}}$ [15].

The addition of SBM to epoxy resin was carried out following the recommendations of manufacturer. Different contents of SBM $(0$, $2.5,5,10 \mathrm{wt} \%$ ) were mixed with epoxy monomer at $120^{\circ} \mathrm{C}$ during 30 min using high-shear mixer (Dispermat) at $2500 \mathrm{rpm}$ to dissolve the triblock copolymer in epoxy resin. Then, the curing agent was added in stoichiometric ratio and the mixture was degassed. The curing process was performed in two steps: $3 \mathrm{~h}$ at $150^{\circ} \mathrm{C}$ and then $1 \mathrm{~h}$ at $180^{\circ} \mathrm{C}$.

\section{Characterization}

The morphology of modified SBM/epoxy resins was studied by Field Emission Gun Scanning Electron Microscopy (FEG-SEM) and Transmission Electron Microscopy (TEM). The samples surfaces were coated in thin films $(40-50 \mathrm{~nm})$ by cryo-microtomy and stained by vapor of $\mathrm{RuO}_{4}$ for their observation by TEM (Phillips Tecnai $200 \mathrm{kV}$ ). On the other hand, the surfaces of the samples were sputter coated by a thin layer $(5-10 \mathrm{~nm})$ of $\mathrm{Au}(\mathrm{Pd})$ for their study by FEG-SEM (Nova NanoSEM FEI 230).

The mechanical characterization was carried out by flexural test (Instron 4465), following the ASTM D-790 at a crosshead speed of 0.8 $\mathrm{mm} / \mathrm{min}$. The fracture surfaces were also covered with $\mathrm{Au}(\mathrm{Pd})$ and observed by and FEG-SEM.

The viability of using these modified epoxy resins as adhesives for joining carbon fiber/epoxy laminates was studied analyzing the joint strength and toughness. The adhesive strength was determined by single lap shear test in accordance with the description given in ASTM D5868. The adherends were $100 \mathrm{~mm}$ long, $25 \mathrm{~mm}$ wide and $2.5 \mathrm{~mm}$ thick. The overlap area of the bonding region was $25 \times 25 \mathrm{~mm}^{2}$ with constant thickness of $0.7 \mathrm{~mm}$. Five specimens were tested for each adhesive composition. Double Cantilever Beam (DCB) tests were performed to determine the mode-I adhesive fracture energy of the adhesive joints, following the protocol "Determination of the Mode-I Adhesive Fracture Energy, $\mathrm{G}_{\mathrm{Ic}}$, of Structural Adhesives using the Double Cantilever Beam (DCB) and Tapered Double Cantilever Beam
(TDCB)" [16]. For this test, the adherends were $150 \mathrm{~mm}$ long, $25 \mathrm{~mm}$ wide and $3.3 \mathrm{~mm}$ thick. A $75 \mathrm{~mm}$ thick Polyethylene Terephthalate (PTFE) film was inserted at one end of the specimen to act as a crack initiator. To perform the test, a pre-crack was generated from the nonadhesive insert, and then the DCB test was performed. The thickness of the adhesive was $0.4 \mathrm{~mm}$. To facilitate the detection of crack growth, one edge of the sample was coated with a thin layer of typewriter correction fluid. In the DCB testing, the load was applied to the specimens at a constant cross-head rate of $1 \mathrm{~mm} / \mathrm{min}$. The measured displacement, $\mathrm{d}$, was corrected with the system compliance $[17,18]$. Five samples were tested for each adhesive composition. There are several methods to calculate the mode-I strain energy release rate, $\mathrm{G}_{\mathrm{Ic}}[18]$ : I) Corrected Beam Theory (CBT); II) Experimental Compliance Method (ECM) and III) Area method. We analyzed the results obtained with each one in a previous work and we have selected the Area method for its simplicity and quality of results in this new research [19]. The procedure of analysis is explained in detail in the published work [18].

\section{Results}

\section{Morphology of SBM/epoxy blends}

The study of phase structure of epoxy resins modified with different contents of SBM block copolymer confirmed the presence of two phases in different scale. Two-phase morphology is one of the most important criteria for enhancement of fracture toughness. Although there are few exceptions, heterogeneous blend gave higher fracture toughness compared with homogeneous blends [9]. Two different electron
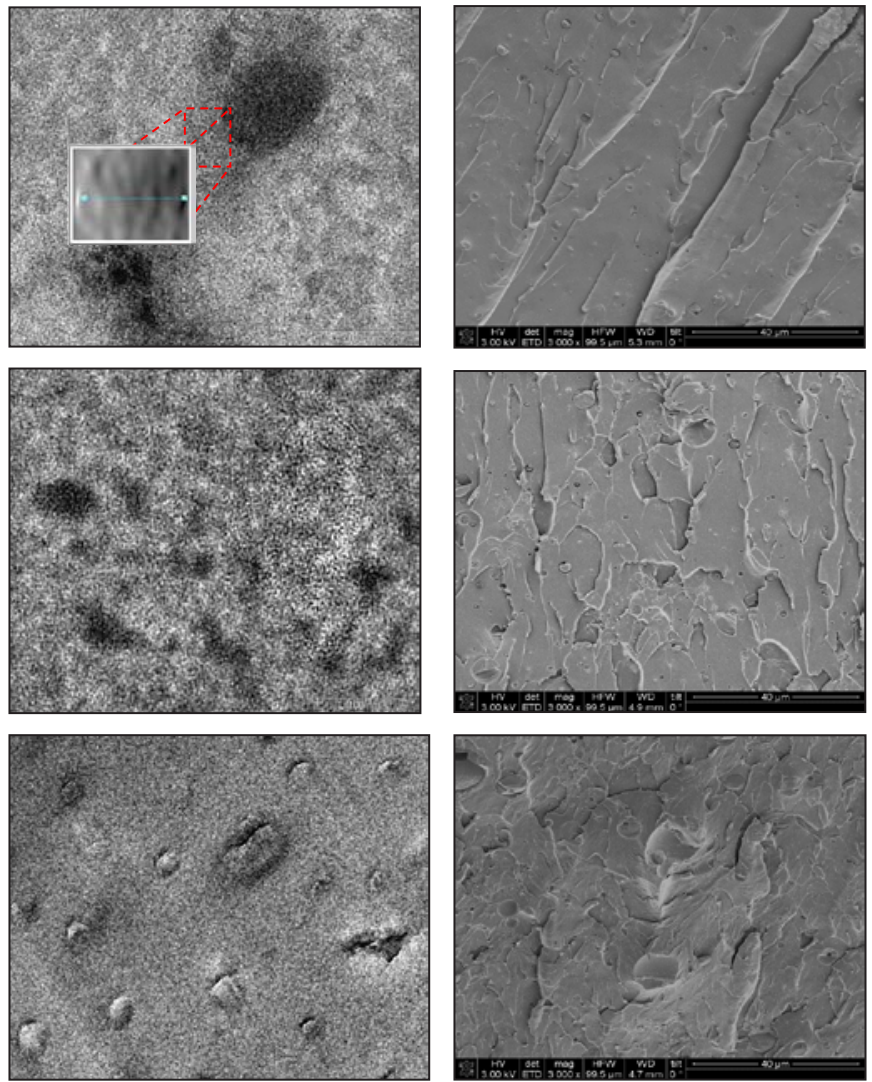

Figure 1: Micrographs obtained by TEM (left) and FEG-SEM (right) of epoxy resins modified with $2.5 \%(1 \mathrm{a}, 1 \mathrm{~b}), 5 \%(1 \mathrm{c}, 1 \mathrm{~d})$ and $10 \%(1 \mathrm{e}, 1 \mathrm{f}) \mathrm{SBM}$ block copolymer. Micrographs $1 \mathrm{a}$ and $1 \mathrm{e}$ shows an image analysis of the nanoinclusions interface. 
Citation: Prolongo SG, Gude MR, Urena A (2012) Adhesive Strength and Toughness Improvement of Epoxy Resin Modified with Polystyrene-BPolybutadiene-B-Poly (Methyl Methacrylate) Block Copolymer. J Material Sci Eng 1:109. doi:10.4172/2169-0022.1000109

microscopies were used in order to analyze both different phases. Figure 1 collects several micrographs captured by FEG-SEM (1b, 1d, 1f) and TEM (1a, 1c, 1e). FEG-SEM micrographs shows spherical micrometer-size particles with an average diameter which varies with the SBM load: $500 \mathrm{~nm}$ for $2.5 \mathrm{wt} \%$ and $1 \mu \mathrm{m}$ for SBM contents of 5 and $10 \mathrm{wt} \%$. In all cases, these particles are broken, indicating a good adhesion between epoxy matrix and dispersed phase. This together with uniform dispersion of domains is also essential for enhancing the fracture toughness [9]. The fracture mechanisms implied will be studied in depth on the tested flexural specimens. In addition to this micro-scale phase, these micrographs show a second phase smaller homogenously distributed into epoxy matrix. In order to determine the size of this phase and even to study their nature and interface, the same samples, stained with ruthenium tetroxide, were observed by TEM. The blends with 2.5 and $5 \mathrm{wt} \%$ SBM present a secondary diffusive phase, whose geometry tends to cylindrical one. This nanoscale phase seems be structured in concentric layers, commonly named "onion like" multilayer morphology [8]. Some authors $[9,11,20,21]$ affirm that the dark centre of nano-inclusions mainly is polybutadiene, which is completely immiscible in epoxy network. The next gray layer, concentric to the PB core, could be mainly formed by polystyrene, whose compatibility with the matrix is lightly higher than polybutadiene. Finally, the PMMA blocks must be soluble in the epoxy matrix. It is worthy to see that the size and shape of nano-phase varies with the amount of SBM added. The epoxy resin modified with $2.5 \%$ SBM shows spherical nanoparticles, whose diameter is close to $50 \mathrm{~nm}$. In contrast, the blend with 5\% SBM shows a cylindrical nano-scale phase showing multilayer morphology. Finally, the resin with highest SBM content presents a different kind of secondary phase. Their structure is not multilayer. The morphology of these nanoinclusions is similar than the micro-scale ones observed by FEG-SEM. They are spherical particles, whose average diameter is around $100 \mathrm{~nm}$, showing a clear and defined interface. So, the epoxy resins modified with 2.5 and $5.0 \mathrm{wt} \% \mathrm{SBM}$ can be regarded as nanostructured epoxy matrix with micro-inclusions dispersed. In contrast, the blend with $10 \%$ block copolymer is formed by two kind of thermoplastic inclusions with different size. This affirmation was confirmed by image analysis (Figure 1a, 1e). It is possible to observe that the nano-scale phase of micrograph 1a, corresponding to epoxy resin modified with $2.5 \mathrm{wt} \%$ SBM does not present a clear interface in contrast to the nano-scale phase of micrograph 1e obtained for epoxy resin modified with $10 \mathrm{wt} \%$ SBM.

\section{Flexural properties of SBM/epoxy blends}

Figure 2 shows the mechanical properties of modified epoxy resins as a function of the percentage of block copolymer added. The epoxy/ SBM blends present enhanced mechanical properties regarding to neat epoxy thermosetting resin. In particular, the addition of low SBM contents (2.5 and $5 \mathrm{wt} \%)$ causes an enhancement on the stiffness and mechanical strength of resin, reaching increases of $24 \%$ and $12 \%$ for the flexural modulus and maximum strength, respectively. The blend reinforced with $10 \%$ SBM present different behavior. The stiffness and strength of this blend are lightly higher than the neat material but it shows an important increase of deformation. The different behavior of blends as a function of SBM load must be associated to the different morphology. In the previous section, it is probed that the phase structure of epoxy resin modified with $10 \%$ SBM is different than the observed one for blends with lower SBM content. In both cases, an increase of the mechanical strength and the deformation ability can induce an increase of toughness, which is our main goal. In order to analyze the possible presence of toughening mechanisms, the fracture surfaces were observed by FEG-SEM (Figure 3). As it is expected, the fracture surfaces of blends with 2.5 and $5.0 \mathrm{wt} \%$ SBM are similar and they present differences regarding to the surface of epoxy resin modified with $10 \mathrm{wt} \% \mathrm{SBM}$. The micrographs of neat epoxy resins have not been included because they have been widely reported in the bibliography since DGEBA/DDM is a common epoxy resin [22,23]. As it is well known, the neat thermosetting resin presents a smooth surface, showing few accentuated fine, straight and parallel river markings, pointing to the defect responsible for the failure, characteristic of a brittle material. In general, the fracture surfaces of blends are rougher, increasing significantly fracture lines concentration as well as some features protruding from the fracture surface usually named "stacked lamellar texture", which imply the fortuitous intersection of two planes of fracture, stating the presence of localized plastic deformation in the matrix. This means that the energy required for the propagation of crack is increased [7]. On the fracture surfaces, it is possible to observe several characteristic features of toughening mechanisms of modified epoxy resins. First of all, an important deformation of matrix is appreciated, appearing cavitations on dispersed thermoplastic micro-scale phase (Figure 3a, 3c, 3e). This toughening mechanism is commonly named "shear yielding" and it is the most frequent on epoxy resins reinforced with a second plastic phase. Plastic deformation blunts the crack tip, reducing the local stress concentration and allowing the material support higher loads before failure occurs. Other toughening mechanism observed is the named "particle bridging". The micro-scale particles deform plastically in the material surrounding the crack tip providing additional crack shielding. It is interesting to note the micro fibrillation formed around the micro-particles (Figure 3a, 3d), named "ductile tearing of particles". Also, no vacant spaces were found due to removal of domains from fracture surface. Both indicate that there exists strong interfacial adhesion between matrix and micro-scale phase due to the preferential solution of some polymeric blocks, such as PMMA, regarding to the immiscibility of PB.

The main differences between fracture surfaces of different blends are observed at high magnification (Figure 3b, 3d, 3f). The secondary nano-scale phase of blends with low SBM content (2.5 and $5 \mathrm{wt} \%$ ) present cavitation and light plastic deformation. In contrast, the nano-inclusions of epoxy resin modified with $10 \mathrm{wt} \%$ SBM present some voids. The debonding process is generally considered to absorb little energy compared to the plastic deformation of matrix. However, debonding is essential because this reduces the constraint at the crack tip and hence allows the matrix to deform plastically via a void growth mechanism [24]. This is the main mechanism which justified the higher deformation ability of this SBM/epoxy blend.

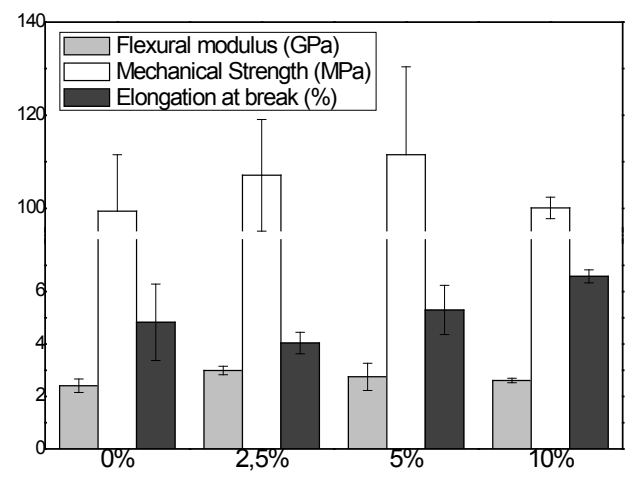

Figure 2: Flexural properties of epoxy resins modified with different SBM load. 
Citation: Prolongo SG, Gude MR, Urena A (2012) Adhesive Strength and Toughness Improvement of Epoxy Resin Modified with Polystyrene-BPolybutadiene-B-Poly (Methyl Methacrylate) Block Copolymer. J Material Sci Eng 1:109. doi:10.4172/2169-0022.1000109
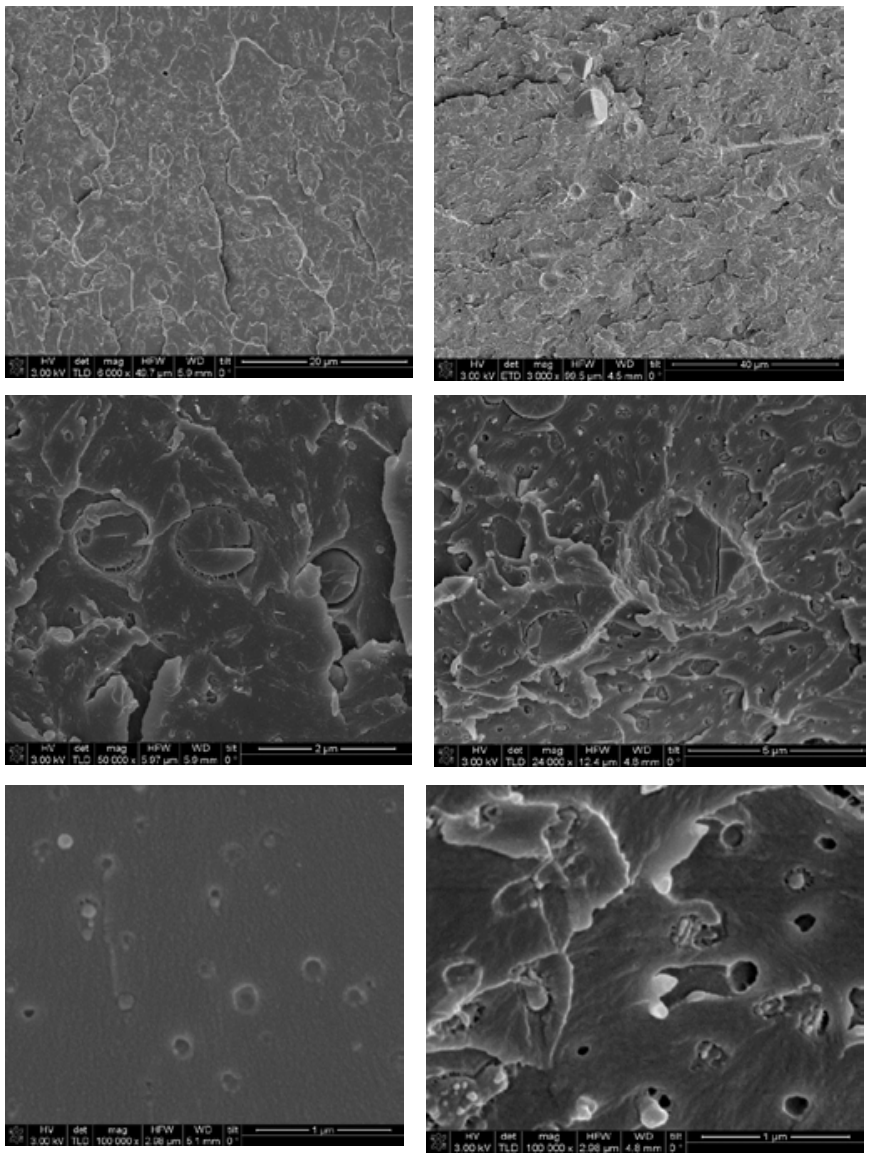

Figure 3: FEG-SEM micrographs of fracture surfaces of epoxy blends modified with 2.5 wt \% (3a, 3b), 5 wt \% (3c, 3d) and 10 wt \% (3e, 3f).

\section{Strength and toughness of carbon fiber/epoxy joints bonded epoxy/SBM adhesives}

In the previous section, it was confirmed that the modification of epoxy resin with SBM induces an important increase of their mechanical properties. Also, the fracture surfaces analysis confirmed the appearance of different toughening mechanisms associated to the presence of two-phase structure. Particularly, the addition of low SBM contents induces an increase of mechanical strength and modulus of epoxy matrix while the incorporation of 10\% SBM implies an increase of deformation ability. In this new section, we analyze how this affects to toughen of epoxy adhesives using epoxy laminates as adherends.

Figure 4 shows the values of lap shear strength and mode-I adhesive fracture energy for the joints bonded with modified epoxy adhesives and unmodified one as a function of weight percentage of SBM added in epoxy resin. The addition of SBM induces an increase of adhesive strength. For a brittle neat epoxy thermosetting with high cohesive strength and low flexibility, the introduction of thermoplastic phases can damp the external stress and enhances the ability of adhesive for resisting against crack propagation [25]. Therefore, the lap shear strength increases. The epoxy resin modified with $10 \mathrm{wt} \%$ SBM presents an enhancement of their adhesive strength of $44 \%$ comparing with neat thermosetting resin. This implies that the increase of deformation ability of brittle epoxy adhesive caused by the addition of $10 \mathrm{wt} \%$ SBM allows enhancing the joint strength. This increase of almost $50 \%$ more on adhesive strength is markedly interesting for future applications. In all cases, the failure mode is adhesive but in the joint bonded with epoxy adhesive modified with $10 \mathrm{wt} \% \mathrm{SBM}$, there were some small areas of cohesive failure in the adherend. This small change in the failure mechanism could indicate a change in the toughness of the joints [18]. For this reason, the toughness of joints was studied by the measurement of mode-I adhesive fracture energy using DCB specimens. In this point, it is necessary to comment that the surface treatment applied on carbon fiber/epoxy laminates is peel ply. Peel ply is very easy to apply and provides rough surfaces, increasing markedly the area available for bonding and therefore the dispersive surface energy of adherend. However, in spite of the higher roughness and improving wetting over untreated adherends, all tested joints bonded with neat epoxy adhesive failed in adhesive mode.

Figure 4 also shows the mode-I adhesive fracture energy values as a function of SBM content of the adhesive. The area method provided one $\mathrm{G}_{\mathrm{Ic}}$ value for each joint. The arithmetic mean and standard deviation were calculated for each adhesive composition using five values of $\mathrm{G}_{\mathrm{Ic}}$ corresponding to the five joints tested. The load-displacement curves obtaining during the DCB testing can present two behaviors [18]: a) the crack progresses continuously, where the curve collected is continuous and smooth without jumps; and b) unstable "stick-slip" crack growth behavior, where load-displacement present numerous small peaks. The crack progressed continuously during the test of joints bonded with neat epoxy adhesive while that the curves collected for all joints bonded with modified epoxy blends exhibited unstable "stick-slip" crack growth. This could indicate that the crack growth on neat adhesive was brittle way easy and fast. In contrast, when joints were bonded with modified epoxy/SBM adhesives, the crack found impediments to progress, which usually implies an increase of toughness. This conclusion is confirmed with $G_{I c}$ values. An important increase of mode-I fracture energy of DCB joints is obtained for the adhesives modified with block copolymers. This increase of adhesive toughness is close to $70 \%$. In spite of the important differences found in the mechanical behaviour of the epoxy blends as a function of SBM content and even in their adhesive strength, not great differences are observed on the fracture energy of their joints. This fact can be associated to the selected method for calculating $G_{I c}$ value, which is easy and reproducible. However, the area method could be less accurate to analyse the toughness of joints which present "stick-slip" crack growth, such as occurs with the modified SBM/epoxy adhesives [18].

The fracture surface of unmodified adhesive is flat whereas a mixed failure mode (cohesive and interfacial) was observed for modified epoxy adhesives. In all studied joints, the initiation zone seems brittle but, in the joints bonded with modified adhesives, there were more signs of

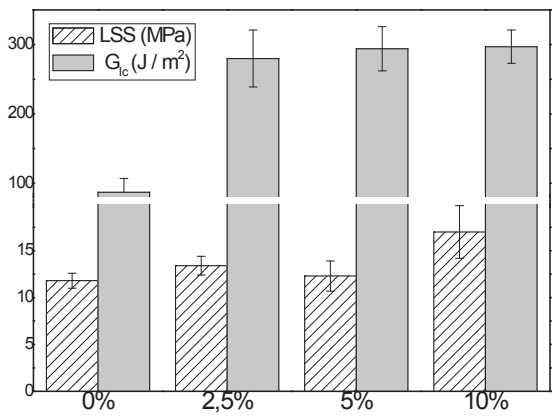

Figure 4: Lap Shear Strength (LSS) and mode-I adhesive fracture energy $\left(\mathrm{G}_{\mathrm{IC}}\right)$ vs. SBM content added to epoxy adhesive. 
Citation: Prolongo SG, Gude MR, Urena A (2012) Adhesive Strength and Toughness Improvement of Epoxy Resin Modified with Polystyrene-BPolybutadiene-B-Poly (Methyl Methacrylate) Block Copolymer. J Material Sci Eng 1:109. doi:10.4172/2169-0022.1000109

microfibrillation, which is a typical mechanism of energy consumption during the fracture of epoxy adhesives.

\section{Conclusions}

The modification of epoxy resin with different contents of polystyrene-b-polybutadiene-b-poly (methyl methacrylate) block copolymer was studied, analyzing their morphology and mechanical properties. Also, the viability of using these modified epoxy resins as adhesives for carbon fiber/epoxy laminates was evaluated, measuring the lap shear strength and mode-I adhesive fracture energy.

The studied blends exhibited a nanostructured epoxy matrix reinforced with micro-scale thermoplastic inclusions. The size and shape of both domains depended on the SBM content and this affected to their mechanical properties. All studied epoxy blends presented enhanced mechanical properties comparing with neat thermosetting resin. At low SBM content (2.5 and $5 \mathrm{wt} \%$ ), the blends presented enhanced flexural modulus and strength while the addition of higher percentage (10 wt\%) implies an important increase of deformation ability. These differences were justified by the appearance of different toughening mechanisms associated to different morphology. In particular, the fracture surface of the resin modified with $10 \mathrm{wt} \% \mathrm{SBM}$ present debonding on nanoscale domains. Although this mechanism generally imply the absorption of little energy, it is essential because this reduces the constraint at the crack tip and hence allows the matrix to deform plastically via a void growth mechanism.

The enhancement of mechanical properties of modified epoxy resins together with their nanostructured morphology caused a dramatic increase of their adhesive properties. The adhesive strength and toughness of joints with carbon fiber/epoxy laminates treated by peel ply were measured. The lap shear adhesive strength is increased up to $50 \%$ regarding to neat epoxy resin and the mode-I adhesive fracture energy $\left(G_{\text {Ic }}\right.$ increased up to $70 \%$. These important enhancements are very significant for future applications taking into account the relative low cost of the block copolymer added and the simplicity and speed of manufacturing process of epoxy resin modified with SBM block copolymer.

\section{Acknowledgements}

Authors wish thank the support of Ministerio de Ciencia e Innovacion of Spain Government (Project MAT2010-207024-C02-01) and Direction General de Universidad of Madrid Community (Project S2009/MAT-1585).

\section{References}

1. Kinloch AJ (2003) Toughening Epoxy Adhesives to Meet Today's Challenges. MRS Bulletin 28: 445-448.

2. Paul DR, Bucknall CB (1999) Polymer Blends: Formulation, John Wiley \& Sons, Inc New York.

3. Kinloch AJ, Yuen ML, Jenkins SD (1994) Thermoplastic-toughened epoxy polymers. J Mater Sci 29: 3781-3790.

4. Pearson RA, Yee AF (1989) Toughening mechanisms in elastomer-modified epoxies. Part 3 The effect of crosslink density. J Mater Sci 24: 2571-2580.

5. Yee AF, Li D, Li X (1993) The importance of constraint relief caused by rubber cavitation in the toughening of epoxy. J Mater Sci 28: 6392-6398.

6. Jordan J, Jacob KI, Tannenbaum R, Sharaf MA, Jasiuk I (2005) Experimental trends in polymer nanocomposites-a review. J Mater Sci 393: 1-11.

7. Ruiz-Perez L, Royston GJ, Fairclough JPA, Ryan AJ (2008) Toughening by nanostructure. Polymer 49: 4475-4488.

8. Kishi H, Kunimitsu Y, Imade J, Oshita S, Morishita Y et al. (2011) Nano-phase structures and mechanical properties of epoxy/acryl triblock copolymer alloys. Polymer 52: 760-768.
9. Francis B, Thomas S, Jose J, Ramaswamy R, Rao VL (2005) Hydroxyl terminated poly(ether etherk etone) with pendent methyl group toughened epoxy resin: miscibility, morphology and mechanical properties. Polymer 46: 12372-12385.

10. Matsushita Y, Suzuki J, Seki M (1998) Surfaces of tricontinuous structure formed by an ABC triblock copolymer in bulk. Phys Rev B 248: 238-242.

11. Fan W, Zheng S (2008) Reaction-induced microphase separation in thermosetting blends of epoxy resin with poly(methyl methacrylate)-blockpolystyrene block copolymers: Effect of topologies of block copolymers on morphological structures. Polymer 49: 3157-3167.

12. Ritzenthaler S, Court F, David L, Girard-Reydet E, Leibler L, et al. (2002) ABC Triblock Copolymers/Epoxy-Diamine Blends. 1. Keys To Achieve Nanostructured Thermosets. Macromolecules 35: 6245-6254.

13. Ritzenthaler S, Court F, Girard-Reydet E, Leibler L, Pascault JP (2003) ABC Triblock Copolymers/Epoxy-Diamine Blends. 2. Parameters Controlling the Morphologies and Properties. Macromolecules 36: 118-126.

14. Ritzenthaler S, Girard-Reydet F, Pascault JP (2000) Influence of epoxy hardener on miscibility of blends of poly(methyl methacrylate) and epoxy networks. Polymer 41: 6375-6386.

15. www.arkema-inc.com

16. Moore DR, Williams JG, Pavan A (2001) Fracture Mechanics Testing Methods for Polymers, Adhesives and Composites. Elsevier Science Ltd UK.

17. Blackman BRK, Kinloch AJ, Paraschi M, Teo WS (2003) Measuring the mode I adhesive fracture energy, $G_{I C}$, of structural adhesive joints: the results of an international round-robin. Int J Adhes Adhes 23: 293-305.

18. Gude MR, Prolongo SG, Gomez-del Rio T, Urena A (2011) Mode-I adhesive fracture energy of carbon fibre composite joints with nanoreinforced epoxy adhesives. Int J Adhes Adhes 31: 695-703.

19. Whitney JM, Browning CE, Hoogsteden W (1982) A Double Cantilever Beam Test for Characterizing Mode I Delamination of Composite Materials. J Rein Plast Comp 1: 297-313.

20. Zucchi IA, Galante MJ, Williams RJJ (2005) Comparison of morphologies and mechanical properties of crosslinked epoxies modified by polystyrene and poly(methyl methacrylate)) or by the corresponding block copolymer polystyrene-b-poly(methyl methacrylate). Polymer 46: 2603-2609.

21. Dean JM, Verghese NE, Pham HQ, Bates FS (2003) Nanostructure Toughened Epoxy Resins. Macromolecules 36: 9267-9270.

22. Prolongo SG, Burón M, Salazar A, Urena A, Rodríguez J (2007) Morphology and dynamic mechanical properties of epoxy/poly(styrene-co-allylalcohol) blends. J Therm Anal Calorim 87: 269-276.

23. Kumar KD, Kothandaraman B (2008) Modification of (DGEBA) epoxy resin with maleated depolymerised natural rubber. eXPRESS Polymer Lett 2: 302-311.

24. Johnsen BB, Kinloch AJ, Mohammed RD, Taylor AC, Sprenger S (2007) Toughening mechanisms of nanoparticle-modified epoxy polymers. Polymer 48: $530-541$

25. Khoee S, Hassani N (2010) Adhesion strength improvement of epoxy resin reinforced with nanoelastomeric copolymer. Mater Sci Eng 527: 6562-6567. 